\title{
Properties of $\theta$-super positive graphs
}

\author{
Cheng Yeaw Ku \\ Department of Mathematics, National University of Singapore, Singapore 117543 \\ matkcy@nus.edu.sg \\ Kok Bin Wong \\ Institute of Mathematical Sciences, University of Malaya, 50603 Kuala Lumpur, Malaysia \\ kbwong@um.edu.my
}

Submitted: Sep 13, 2011; Accepted: Jan 26, 2012; Published: Feb 7, 2012

Mathematics Subject Classifications: 05C31, 05C70

\begin{abstract}
Let the matching polynomial of a graph $G$ be denoted by $\mu(G, x)$. A graph $G$ is said to be $\theta$-super positive if $\mu(G, \theta) \neq 0$ and $\mu(G \backslash v, \theta)=0$ for all $v \in V(G)$. In particular, $G$ is 0 -super positive if and only if $G$ has a perfect matching. While much is known about 0 -super positive graphs, almost nothing is known about $\theta$ super positive graphs for $\theta \neq 0$. This motivates us to investigate the structure of $\theta$-super positive graphs in this paper. Though a 0 -super positive graph need not contain any cycle, we show that a $\theta$-super positive graph with $\theta \neq 0$ must contain a cycle. We introduce two important types of $\theta$-super positive graphs, namely $\theta$ elementary and $\theta$-base graphs. One of our main results is that any $\theta$-super positive graph $G$ can be constructed by adding certain type of edges to a disjoint union of $\theta$-base graphs; moreover, these $\theta$-base graphs are uniquely determined by $G$. We also give a characterization of $\theta$-elementary graphs: a graph $G$ is $\theta$-elementary if and only if the set of all its $\theta$-barrier sets form a partition of $V(G)$. Here, $\theta$-elementary graphs and $\theta$-barrier sets can be regarded as $\theta$-analogue of elementary graphs and Tutte sets in classical matching theory.
\end{abstract}

KEYWORDS: matching polynomial, Gallai-Edmonds decomposition, elementary graph, barrier sets, extreme sets 


\section{Introduction}

We begin by introducing matching polynomials with an interest in the multiplicities of their roots. This will lead us to a recent extension of the celebrated Gallai-Edmonds Strcuture Theorem by Chen and $\mathrm{Ku}$ [1] which will be useful later in our study of $\theta$-super positive graphs. This result has been instrumental in recent investigations of the subject, see $[5,6,7,8,9,10]$.

All the graphs in this paper are simple and finite. The vertex set and edge set of a graph $G$ will be denoted by $V(G)$ and $E(G)$, respectively.

Definition 1.1. An $r$-matching in a graph $G$ is a set of $r$ edges, no two of which have a vertex in common. The number of $r$-matchings in $G$ will be denoted by $p(G, r)$. We set $p(G, 0)=1$ and define the matching polynomial of $G$ by

$$
\mu(G, x)=\sum_{r=0}^{\lfloor n / 2\rfloor}(-1)^{r} p(G, r) x^{n-2 r}
$$

where $n=|V(G)|$. We denote the multiplicity of $\theta$ as a root of $\mu(G, x)$ by $\operatorname{mult}(\theta, G)$. Let $u \in V(G)$, the graph obtained from $G$ by deleting the vertex $u$ and all edges that contain $u$ is denoted by $G \backslash u$. Inductively if $u_{1}, \ldots, u_{k} \in V(G), G \backslash u_{1} \cdots u_{k}=\left(G \backslash u_{1} \cdots u_{k-1}\right) \backslash u_{k}$. Note that the order in which the vertices are being deleted is not important, that is, if $i_{1}, \ldots, i_{k}$ is a permutation of $1, \ldots, k$, we have $G \backslash u_{1} \cdots u_{k}=G \backslash u_{1_{1}} \cdots u_{i_{k}}$. Furthermore, if $X=\left\{u_{1}, \ldots, u_{k}\right\}$, we set $G \backslash X=G \backslash u_{1} \cdots u_{k}$. If $H$ is a subgraph of $G$, by an abuse of notation, we have $G \backslash H=G \backslash V(H)$. For example, if $p=v_{1} v_{2} \ldots v_{n}$ is a path in $G$ then $G \backslash p=G \backslash v_{1} v_{2} \cdots v_{n}$. If $e$ is an edge of $G$, let $G-e$ denote the graph obtained from $G$ by deleting the edge $e$ from $G$. Inductively, if $e_{1}, \ldots, e_{k} \in E(G)$, $G-e_{1} \cdots e_{k}=\left(G-e_{1} \cdots e_{k-1}\right)-e_{k}$.

A graph $G$ is said to have a perfect matching if it has an $n / 2$-matching ( $n$ must be even). This is equivalent to $\operatorname{mult}(0, G)=0$, that is, 0 is not a root of $\mu(G, x)$. Recall that in the literature mult $(0, G)$ is also known as the deficiency of $G$ which is the number of vertices of $G$ missed by some maximum matching.

The following are some basic properties of $\mu(G, x)$.

Theorem 1.2. [2, Theorem 1.1 on p. 2]

(a) $\mu(G \cup H, x)=\mu(G, x) \mu(H, x)$ where $G$ and $H$ are disjoint graphs,

(b) $\mu(G, x)=\mu(G-e, x)-\mu(G \backslash u v, x)$ if $e=(u, v)$ is an edge of $G$,

(c) $\mu(G, x)=x \mu(G \backslash u, x)-\sum_{i \sim u} \mu(G \backslash u i, x)$ where $i \sim u$ means $i$ is adjacent to $u$,

(d) $\frac{d}{d x} \mu(G, x)=\sum_{i \in V(G)} \mu(G \backslash i, x)$ where $V(G)$ is the vertex set of $G$. 
It is well known that all roots of $\mu(G, x)$ are real. Throughout, let $\theta$ be a real number. The multiplicity of a matching polynomial root satisfies the the following interlacing property:

Lemma 1.3. [2, Corollary 1.3 on p. 97] (Interlacing) Let $G$ be a graph and $u \in V(G)$. Let $\theta$ be a real number. Then

$$
\operatorname{mult}(\theta, G)-1 \leq \operatorname{mult}(\theta, G \backslash u) \leq \operatorname{mult}(\theta, G)+1 .
$$

Lemma 1.3 suggests that given any real number $\theta$, we can classify the vertices of a graph according to an increase of 1 or a decrease of 1 or no change in the multiplicity of $\theta$ upon deletion of a vertex.

Definition 1.4. [3, Section 3] For any $u \in V(G)$,

(a) $u$ is $\theta$-essential if mult $(\theta, G \backslash u)=\operatorname{mult}(\theta, G)-1$,

(b) $u$ is $\theta$-neutral if $\operatorname{mult}(\theta, G \backslash u)=\operatorname{mult}(\theta, G)$,

(c) $u$ is $\theta$-positive if $\operatorname{mult}(\theta, G \backslash u)=\operatorname{mult}(\theta, G)+1$.

Furthermore, if $u$ is not $\theta$-essential but it is adjacent to some $\theta$-essential vertex, we say $u$ is $\theta$-special.

It turns out that $\theta$-special vertices play an important role in the Gallai-Edmonds Decomposition of a graph (see [1]). Godsil [3, Corollary 4.3] proved that a $\theta$-special vertex must be $\theta$-positive. Note that if $\operatorname{mult}(\theta, G)=0$ then for any $u \in V(G), u$ is either $\theta$-neutral or $\theta$-positive and no vertices in $G$ can be $\theta$-special. Now $V(G)$ can be partitioned into the following sets:

$$
V(G)=D_{\theta}(G) \cup A_{\theta}(G) \cup P_{\theta}(G) \cup N_{\theta}(G),
$$

where

$D_{\theta}(G)$ is the set of all $\theta$-essential vertices in $G$,

$A_{\theta}(G)$ is the set of all $\theta$-special vertices in $G$,

$N_{\theta}(G)$ is the set of all $\theta$-neutral vertices in $G$,

$P_{\theta}(G)=Q_{\theta}(G) \backslash A_{\theta}(G)$, where $Q_{\theta}(G)$ is the set of all $\theta$-positive vertices in $G$.

Note that there are no 0-neutral vertices. So $N_{0}(G)=\varnothing$ and $V(G)=D_{0}(G) \cup A_{0}(G) \cup$ $P_{0}(G)$. 
Definition 1.5. [3, Section 3] A graph $G$ is said to be $\theta$-critical if all vertices in $G$ are $\theta$-essential and $\operatorname{mult}(\theta, G)=1$.

The celebrated Gallai-Edmonds Structure Theorem describes the stability of a certain canonical decomposition of $V(G)$ with respect to the zero root of $\mu(G, x)$. In [1], Chen and $\mathrm{Ku}$ extended the Gallai-Edmonds Structure Theorem to any root $\theta \neq 0$, which consists of the following two theorems:

Theorem 1.6. [1, Theorem 1.5] ( $\theta$-Stability Lemma) Let $G$ be a graph with $\theta$ a root of $\mu(G, x)$. If $u \in A_{\theta}(G)$ then

(i) $D_{\theta}(G \backslash u)=D_{\theta}(G)$,

(ii) $P_{\theta}(G \backslash u)=P_{\theta}(G)$,

(iii) $N_{\theta}(G \backslash u)=N_{\theta}(G)$,

(iv) $A_{\theta}(G \backslash u)=A_{\theta}(G) \backslash\{u\}$.

Theorem 1.7. [1, Theorem 1.7] ( $\theta$-Gallai's Lemma) If $G$ is connected and every vertex of $G$ is $\theta$-essential then $\operatorname{mult}(\theta, G)=1$.

Theorem 1.6 asserts that the decomposition of $V(G)$ into $D_{\theta}(G), P_{\theta}(G), N_{\theta}(G)$ and $A_{\theta}(G)$ is stable upon deleting a $\theta$-special vertex of $G$. We may delete every such vertex one by one until there are no $\theta$-special vertices left. Together with Theorem 1.7 , it is not hard to deduce the following whose proof is omitted.

\section{Corollary 1.8.}

(i) $A_{\theta}\left(G \backslash A_{\theta}(G)\right)=\varnothing, D_{\theta}\left(G \backslash A_{\theta}(G)\right)=D_{\theta}(G), P_{\theta}\left(G \backslash A_{\theta}(G)\right)=P_{\theta}(G)$, and $N_{\theta}(G \backslash$ $\left.A_{\theta}(G)\right)=N_{\theta}(G)$.

(ii) $G \backslash A_{\theta}(G)$ has exactly $\left|A_{\theta}(G)\right|+\operatorname{mult}(\theta, G) \theta$-critical components.

(iii) If $H$ is a component of $G \backslash A_{\theta}(G)$ then either $H$ is $\theta$-critical or mult $(\theta, H)=0$.

(iv) The subgraph induced by $D_{\theta}(G)$ consists of all the $\theta$-critical components in $G \backslash A_{\theta}(G)$.

This paper is devoted to the study of $\theta$-super positive graphs. A graph is $\theta$-super positive if $\theta$ is not a root of $\mu(G, x)$ but is a root of $\mu(G \backslash v, x)$ for every $v \in V(G)$. It is worth noting that $G$ is 0 -super positive if and only if $G$ has a perfect matching. While much is known about graphs with a perfect matching, almost nothing is known about $\theta$-super positive graphs for $\theta \neq 0$. This gives us a motivation to investigate the structure of these graphs.

The outline of this paper is as follows: 
In Section 2, we show how to construct $\theta$-super positive graphs from smaller $\theta$-super positive graphs (see Theorem 2.2). We prove that a tree is $\theta$-super positive if and only if $\theta=0$ and it has a perfect matching (see Theorem 2.4). Consequently, a $\theta$-super positive graph must contain a cycle when $\theta \neq 0$. For a connected vertex transitive graph $G$, we prove that it is $\theta$-super positive for any root $\theta$ of $\mu(G \backslash v, x)$ where $v \in V(G)$ (see Theorem 2.8). Finally we prove that if $G$ is $\theta$-super positive, then $N_{\theta}(G \backslash v)=\varnothing$ for all $v \in V(G)$ (see Theorem 2.9).

In Section 3, we introduce $\theta$-elementary graphs. These are $\theta$-super positive graphs with $P_{\theta}(G \backslash v)=\emptyset$ for all $v \in V(G)$. We prove a characterization of $\theta$-elementary graphs: a graph $G$ is $\theta$-elementary if and only if the set of all $\theta$-barrier sets form a partition of $V(G)$ (see Theorem 3.13).

In Section 4, we apply our results in Section 3 to prove that an $n$-cycle $C_{n}$ is 1 elementary if and only if $n=3 k$ for some $k \in \mathbb{N}$ (see Theorem 4.4). Furthermore, we prove that $C_{3 k}$ has exactly 31 -barrier sets (see Corollary 4.5 ).

In Section 5, we introduce $\theta$-base graphs which can be regarded as building blocks of $\theta$-super positive graphs. We prove a characterization of $\theta$-super positive graphs, namely a $\theta$-super positive graph can be constructed from a disjoint union of $\theta$-base graphs by adding certain type of edges; moreover, these $\theta$-base graphs are uniquely determined by $G$ (see Theorem 5.7 and Corollary 5.9).

\section{$2 \quad \theta$-super positive graphs}

Definition 2.1. A graph $G$ is $\theta$-super positive if $\theta$ is not a root of $\mu(G, x)$ and every vertex of $G$ is $\theta$-positive.

By Lemma 1.3, this is equivalent to $\operatorname{mult}(\theta, G)=0$ and $\operatorname{mult}(\theta, G \backslash v)=1$ for all $v \in V(G)$. There are a lot of $\theta$-super positive graphs. For instance the three cycle $C_{3}$ and the six cycle $C_{6}$ are 1 -super positive. In the next theorem, we will show how to construct $\theta$-super positive graphs from smaller $\theta$-super positive graphs.

Theorem 2.2. Let $G_{1}$ and $G_{2}$ be two $\theta$-super positive graphs and $v_{i} \in V\left(G_{i}\right)$ for $i=1,2$. Let $G$ be the graph obtained by adding the edge $\left(v_{1}, v_{2}\right)$ to the union of $G_{1}$ and $G_{2}$. Then $G$ is $\theta$-super positive.

Proof. Let $e=\left(v_{1}, v_{2}\right)$. First we prove that $\mu(G, \theta) \neq 0$. By part (b) of Theorem 1.2, we have $\mu(G, x)=\mu(G-e, x)-\mu\left(G \backslash v_{1} v_{2}, x\right)$. It then follows from part (a) of Theorem 1.2 that $\mu(G, x)=\mu\left(G_{1}, x\right) \mu\left(G_{2}, x\right)-\mu\left(G_{1} \backslash v_{1}, x\right) \mu\left(G_{2} \backslash v_{2}, x\right)$. Since $G_{1}$ and $G_{2}$ are $\theta$-super positive, $\mu(G, \theta)=\mu\left(G_{1}, \theta\right) \mu\left(G_{2}, \theta\right) \neq 0$.

It is left to prove that $\mu(G \backslash v, \theta)=0$ for all $v \in V(G)$. Let $v \in V\left(G_{1}\right)$. Suppose $v=v_{1}$. Then by part (a) of Theorem 1.2, $\mu(G \backslash v, x)=\mu\left(G_{1} \backslash v_{1}, x\right) \mu\left(G_{2}, x\right)$, and 
thus $\mu(G \backslash v, \theta)=0$. Suppose $v \neq v_{1}$. By part (b) of Theorem 1.2, $\mu(G \backslash v, x)=$ $\mu((G \backslash v)-e, x)-\mu\left((G \backslash v) \backslash v_{1} v_{2}, x\right)$. Note that $(G \backslash v)-e=\left(G_{1} \backslash v\right) \cup G_{2}$ and $(G \backslash v) \backslash v_{1} v_{2}=$ $\left(G_{1} \backslash v v_{1}\right) \cup\left(G_{2} \backslash v_{2}\right)$. Hence $\mu(G \backslash v, \theta)=\mu\left(G_{1} \backslash v, \theta\right) \mu\left(G_{2}, \theta\right)-\mu\left(G_{1} \backslash v v_{1}, \theta\right) \mu\left(G_{2} \backslash v_{2}, \theta\right)=0$ (part (a) of Theorem 1.2).

The case $v \in V\left(G_{2}\right)$ is proved similarly.

The graph $G$ in Figure 1 is constructed by using Theorem 2.2, with $G_{1}=C_{6}$ and $G_{2}=C_{3}$. Therefore it is 1-super positive graph.

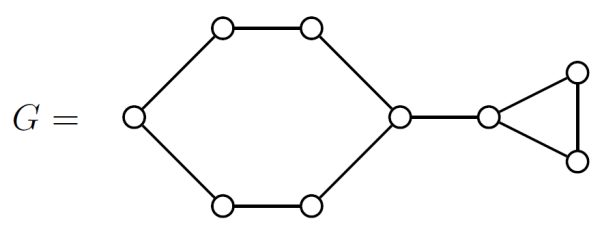

Figure 1.

It is clear that a 0 -super positive need not contain any cycle. However, we will show later that if $G$ is $\theta$-super positive and $\theta \neq 0$, then it must contain a cycle (see Corollary 2.5). Note that any tree $T$ with at least three vertices can be represented in the following form (see Figure 2), where $u$ is a vertex with $n+1$ neighbors $v_{1}, \ldots, v_{n+1}$ such that all of them except possibly $v_{1}$ have degree 1 and $T_{1}$ is a subtree of $T$ that contains $v_{1}$. Such a representation of $T$ is denoted by $\left(T_{1}, u ; v_{1}, \ldots, v_{n+1}\right)$.

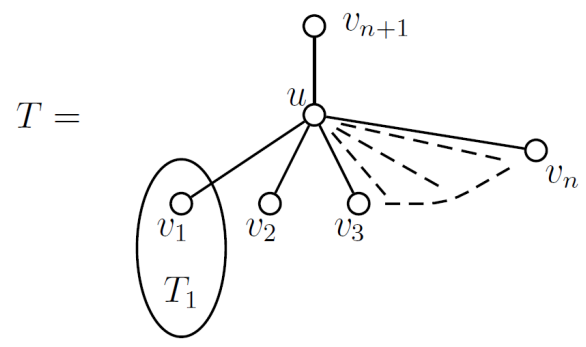

Figure 2.

Lemma 2.3. Let $T$ be a tree with at least three vertices. Suppose $T$ has a representation $\left(T_{1}, u ; v_{1}, \ldots, v_{n+1}\right)$. Then $\theta$ is a root of $\mu(T, x)$ if and only if

$$
\left(n-\theta^{2}\right) \theta^{n-1} \mu\left(T_{1}, \theta\right)+\theta^{n} \mu\left(T_{1} \backslash v_{1}, \theta\right)=0 .
$$

Proof. By part (c) of Theorem 1.2, $\mu(T, \theta)=\theta \mu(T \backslash u, \theta)-\sum_{i=1}^{n+1} \mu\left(T \backslash u v_{i}, \theta\right)$ (see Figure 2), which implies (using part (a) of Theorem 1.2),

$$
\mu(T, \theta)=\left(\theta^{2}-n\right) \theta^{n-1} \mu\left(T_{1}, \theta\right)-\theta^{n} \mu\left(T_{1} \backslash v_{1}, \theta\right) .
$$

Hence the lemma holds. 
Theorem 2.4. Let $T$ be a tree. Then $T$ is $\theta$-super positive if and only if $\theta=0$ and it has a perfect matching.

Proof. Suppose $T$ is $\theta$-super positive and $\theta \neq 0$. Then $T$ must have at least three vertices. By Lemma 2.3,

$$
\left(n-\theta^{2}\right) \theta^{n-1} \mu\left(T_{1}, \theta\right)+\theta^{n} \mu\left(T_{1} \backslash v_{1}, \theta\right) \neq 0 .
$$

By part (a) of Theorem 1.2, $0=\mu(T \backslash u, \theta)=\theta^{n} \mu\left(T_{1}, \theta\right)$ (see Figure 2). Therefore $\mu\left(T_{1}, \theta\right)=0$ and $\mu\left(T_{1} \backslash v_{1}, \theta\right) \neq 0$. Now $\mu\left(T \backslash v_{n+1}, \theta\right)=0$. By part (c) of of Theorem 1.2, $\mu\left(T \backslash v_{n+1}, \theta\right)=\theta \mu\left(T \backslash u v_{n+1}, \theta\right)-\sum_{i=1}^{n} \mu\left(T \backslash u v_{i} v_{n+1}, \theta\right)=\theta^{n} \mu\left(T_{1}, \theta\right)-(n-1) \theta^{n-2} \mu\left(T_{1}, \theta\right)-$ $\theta^{n-1} \mu\left(T_{1} \backslash v_{1}, \theta\right)$. This implies that $\mu\left(T_{1} \backslash v_{1}, \theta\right)=0$, a contradiction. Hence $\theta=0$. Since 0 is not a root of $\mu(T, x), T$ must have a perfect matching.

The converse is obvious.

A consequence of Theorem 2.4 is the following corollary.

Corollary 2.5. If $G$ is $\theta$-super positive for some $\theta \neq 0$, then $G$ must contain a cycle.

We shall need the following lemmas.

Lemma 2.6. [4, Theorem 6.3](Heilmann-Lieb Identity) Let $u, v \in V(G)$. Then

$$
\mu(G \backslash u, x) \mu(G \backslash v, x)-\mu(G, x) \mu(G \backslash u v)=\sum_{p \in \mathcal{P}(u, v)} \mu(G \backslash p, x)^{2},
$$

where $\mathcal{P}(u, v)$ is the set of all the paths from $u$ to $v$ in $G$.

Lemma 2.7. [3, Lemma 3.1] Suppose $\operatorname{mult}(\theta, G)>0$. Then $G$ contains at least one $\theta$-essential vertex.

Theorem 2.8. Let $G$ be connected, vertex transitive and $z \in V(G)$. If $\theta$ is a root of $\mu(G \backslash z, x)$ then $G$ is $\theta$-super positive.

Proof. Since $G \backslash z$ is isomorphic to $G \backslash y$ for all $y \in V(G), \mu(G \backslash z, x)=\mu(G \backslash y, x)$ for all $y \in V(G)$. So $\operatorname{mult}(\theta, G \backslash z)=\operatorname{mult}(\theta, G \backslash y)$. This implies that $\theta$ is a root of $\mu(G \backslash y, x)$ for all $y$.

Now it remains to show that $\mu(G, \theta) \neq 0$. Suppose the contrary. Then by Lemma 2.7, $G$ has at least one $\theta$-essential vertex. Since $G$ is vertex transitive, all vertices in $G$ are $\theta$-essential. By Theorem 1.7, $\operatorname{mult}(\theta, G)=1$. But then mult $(\theta, G \backslash z)=0$, a contradiction. Hence $\mu(G, \theta) \neq 0$ and $G$ is $\theta$-super positive.

However, a $\theta$-super positive graph is not necessarily vertex transitive (see Figure 1 ). Furthermore a $\theta$-super positive graph is not necessary connected, for the union of two $C_{3}$ is 1-super positive. 
Theorem 2.9. Let $G$ be $\theta$-super positive. Then $N_{\theta}(G \backslash v)=\varnothing$ for all $v \in V(G)$.

Proof. Suppose $N_{\theta}(G \backslash v) \neq \varnothing$ for some $v \in V(G)$. Let $u \in N_{\theta}(G \backslash v)$. By Lemma 2.6,

$$
\mu(G \backslash u, x) \mu(G \backslash v, x)-\mu(G, x) \mu(G \backslash u v)=\sum_{p \in \mathcal{P}(u, v)} \mu(G \backslash p, x)^{2} .
$$

Note that the multiplicity of $\theta$ as a root of $\mu(G \backslash u, x) \mu(G \backslash v, x)$ is 2, while the multiplicity of $\theta$ as a root of $\mu(G, x) \mu(G \backslash v u, x)$ is 1 since $u$ is $\theta$-neutral in $G \backslash v$. Therefore the multiplicity of $\theta$ as a root of the polynomial on the left-hand side of the equation is at least 1 . But the multiplicity of $\theta$ as a root of the polynomial on the right-hand side of the equation is even and so, in comparison with the left-hand side, it must be at least 2. This forces the multiplicity of $\theta$ as a root of $\mu(G, x) \mu(G \backslash v u, x)$ to be at least 2, a contradiction. Hence $N_{\theta}(G \backslash v)=\varnothing$ for all $v \in V(G)$.

Now we know that for a $\theta$-super positive graph $G, N_{\theta}(G \backslash v)=\varnothing$ for all $v \in V(G)$. So it is quite natural to ask whether $P_{\theta}(G \backslash v)=\varnothing$ for all $v \in V(G)$. Well, this is not true in general (see Figure 1). This motivates us to study the $\theta$-super positive graph $G$, for which $P_{\theta}(G \backslash v)=\varnothing$ for all $v \in V(G)$. We proceed to do this in the next section.

\section{$3 \quad \theta$-elementary graphs}

Definition 3.1. A graph $G$ is said to be $\theta$-elementary if it is $\theta$-super positive and $P_{\theta}(G \backslash$ $v)=\varnothing$ for all $v \in V(G)$.

The graph $G$ in Figure 3 is 1 -elementary. Not every $\theta$-positive graph is $\theta$-elementary. For instance, the graph in Figure 1 is not 1-elementary.

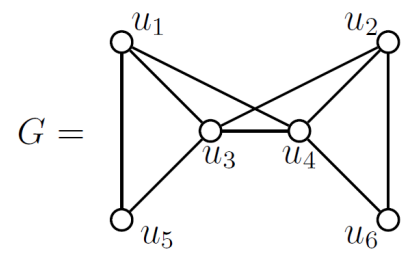

Figure 3.

Theorem 3.2. A graph $G$ is $\theta$-elementary if and only if $\operatorname{mult}(\theta, G)=0$ and $P_{\theta}(G \backslash v) \cup$ $N_{\theta}(G \backslash v)=\varnothing$ for all $v \in V(G)$.

Proof. Suppose $\operatorname{mult}(\theta, G)=0$ and $P_{\theta}(G \backslash v) \cup N_{\theta}(G \backslash v)=\varnothing$ for all $v \in V(G)$. For each $v \in V(G), G \backslash v$ must consist entirely of $\theta$-essential and $\theta$-special vertices and must have at least one $\theta$-essential vertex; therefore $\operatorname{mult}(\theta, G \backslash v)=1$. Therefore $G$ is $\theta$-super positive and it is $\theta$-elementary.

The other implication follows from Theorem 2.9. 
It turns out that the notion of a 0-elementary graph coincide with the classical notion of an elementary graph. Properties of elementary graphs can be found in Section 5.1 on p. 145 of [11].

The number of $\theta$-critical components in $G$ is denoted by $c_{\theta}(G)$.

Definition 3.3. A $\theta$-barrier set is defined to be a set $X \subseteq V(G)$ for which $c_{\theta}(G \backslash X)=$ $\operatorname{mult}(\theta, G)+|X|$.

A $\theta$-extreme set in $G$ is defined to be a set $X \subseteq V(G)$ for which $\operatorname{mult}(\theta, G \backslash X)=$ $\operatorname{mult}(\theta, G)+|X|$.

$\theta$-barrier sets and $\theta$-extreme sets can be regarded as $\theta$-analogue of Tutte sets and extreme sets in classical matching theory. Properties of $\theta$-barrier sets and $\theta$-extreme sets have been studied by $\mathrm{Ku}$ and Wong [5]. In particular, the following results are needed.

Lemma 3.4. [5, Lemma 2.4] A subset of a $\theta$-extreme set is a $\theta$-extreme set.

Lemma 3.5. [5, Lemma 2.5] If $X$ is a $\theta$-barrier set in $G$ and $Y \subseteq X$ then $X \backslash Y$ is a $\theta$-barrier set in $G \backslash Y$.

Lemma 3.6. [5, Lemma 2.6] Every $\theta$-extreme set in $G$ lies in a $\theta$-barrier set in $G$.

Lemma 3.7. [5, Lemma 2.7] Let $X$ be a $\theta$-barrier set in $G$. Then $X$ is a $\theta$-extreme set in $G$.

Lemma 3.8. [5, Lemma 3.1] If $X$ is a $\theta$-barrier set in $G$ then $X \subseteq A_{\theta}(G) \cup P_{\theta}(G)$.

Lemma 3.9. [5, Theorem 3.5] Let $X$ be a $\theta$-barrier set in $G$. Then $A_{\theta}(G) \subseteq X$.

Lemma 3.10. Let $G$ be a graph. If $X$ is a $\theta$-barrier set in $G, x \in X$ and $P_{\theta}(G \backslash x)=\varnothing$, then $A_{\theta}(G \backslash x)=X \backslash\{x\}$.

Proof. By Lemma 3.5, $X \backslash\{x\}$ is a $\theta$-barrier set in $G \backslash x$. By Lemma 3.8, $X \backslash\{x\} \subseteq$ $A_{\theta}(G \backslash x) \cup P_{\theta}(G \backslash x)$. Therefore $X \backslash\{x\} \subseteq A_{\theta}(G \backslash x)$. It then follows from Lemma 3.9 that $A_{\theta}(G \backslash x)=X \backslash\{x\}$.

Definition 3.11. We define $\mathfrak{P}(\theta, G)$ to be the set of all the $\theta$-barrier sets in $G$.

Note that in Figure 3, $\mathfrak{P}(1, G)=\left\{\left\{u_{1}\right\},\left\{u_{2}\right\},\left\{u_{3}, u_{4}\right\},\left\{u_{5}\right\},\left\{u_{6}\right\}\right\}$. Now Lemma 3.12 follows from part (c) of Theorem 1.2.

Lemma 3.12. Suppose $G$ is $\theta$-super positive. Then for each $v \in V(G)$ there is a $u \in V(G)$ with $(u, v) \in E(G)$ and $\operatorname{mult}(\theta, G \backslash u v)=0$.

Theorem 3.13. A graph $G$ is $\theta$-elementary if and only if $\mathfrak{P}(\theta, G)$ is a partition of $V(G)$. 
Proof. Let $\mathfrak{P}(\theta, G)=\left\{S_{1}, \ldots, S_{k}\right\}$.

$(\Rightarrow)$ Suppose $G$ is $\theta$-elementary. Then for each $v \in V(G),\{v\}$ is a $\theta$-extreme set. By Lemma 3.6, it is contained in some $\theta$-barrier set. Therefore $V(G)=S_{1} \cup \cdots \cup S_{k}$. It remains to prove that $S_{i} \cap S_{j}=\varnothing$ for $i \neq j$. Suppose the contrary. Let $x \in S_{i} \cap S_{j}$. By Lemma 3.10, $S_{i} \backslash\{x\}=A_{\theta}(G \backslash x)=S_{j} \backslash\{x\}$ and so $S_{i}=S_{j}$, a contradiction. Hence $S_{i} \cap S_{j}=\varnothing$ for $i \neq j$ and $\mathfrak{P}(\theta, G)$ is a partition of $V(G)$.

$(\Leftarrow)$ Suppose $\mathfrak{P}(\theta, G)$ is a partition of $V(G)$. Let $v \in V(G)$. Then $v \in S_{i}$ for some $\theta$ barrier set $S_{i}$. By Lemma 3.8, $v \in A_{\theta}(G) \cup P_{\theta}(G)$. Therefore $V(G) \subseteq A_{\theta}(G) \cup P_{\theta}(G)$. This implies that $\operatorname{mult}(\theta, G)=0$, for otherwise $D_{\theta}(G) \neq \varnothing$ by Lemma 2.7. Hence $A_{\theta}(G)=\varnothing$ and $V(G)=P_{\theta}(G)$, i.e., $G$ is $\theta$-super positive. It remains to show that $P_{\theta}(G \backslash v)=\varnothing$ for all $v \in V(G)$. Suppose the contrary. Then $P_{\theta}\left(G \backslash v_{0}\right) \neq \varnothing$ for some $v_{0} \in V(G)$. We may assume $v_{0} \in S_{1}$. By Corollary 1.8, $\left(G \backslash v_{0}\right) \backslash A_{\theta}\left(G \backslash v_{0}\right)$ has a component $H$ for which $\operatorname{mult}(\theta, H)=0$. By Theorem 2.9, $N_{\theta}\left(G \backslash v_{0}\right)=\varnothing$. So we conclude that $H$ is $\theta$-super positive. Let $w \in H$. By Lemma 3.12, there is a $z \in V(H)$ with $(w, z) \in E(H)$ and $\operatorname{mult}(\theta, H \backslash w z)=0$. By part (a) of Theorem 1.2, and, (ii) and (iii) of Corollary 1.8, $\operatorname{mult}\left(\theta,\left(\left(G \backslash v_{0}\right) \backslash A_{\theta}\left(G \backslash v_{0}\right)\right) \backslash w z\right)=1+\left|A_{\theta}\left(G \backslash v_{0}\right)\right|$.

On the other hand, by Lemma 3.5, $S_{1} \backslash\left\{v_{0}\right\}$ is a $\theta$-barrier set in $G \backslash v_{0}$. So by Lemma 3.9, $A_{\theta}\left(G \backslash v_{0}\right) \subseteq S_{1} \backslash\left\{v_{0}\right\}$. By Lemma 3.5 again, $S_{1} \backslash\left(\left\{v_{0}\right\} \cup A_{\theta}\left(G \backslash v_{0}\right)\right)$ is a $\theta$-barrier set in $\left(G \backslash v_{0}\right) \backslash A_{\theta}\left(G \backslash v_{0}\right)$. Note that $w$ is $\theta$-positive in $G \backslash v_{0}$ (by Corollary 1.8). Therefore $\left\{w, v_{0}\right\}$ is an $\theta$-extreme set in $G$. By Lemma 3.6, $\left\{w, v_{0}\right\}$ is contained in some $\theta$-barrier set in $G$. Since $\mathfrak{P}(\theta, G)$ is a partition of $V(G)$ and $v_{0} \in S_{1}$, we must have $\left\{w, v_{0}\right\} \subseteq S_{1}$. Note also $z$ is $\theta$-positive in $G \backslash v_{0}$ (recall that $H$ is $\theta$-super positive). Using a similar argument, we can show that $\left\{z, v_{0}\right\} \subseteq S_{1}$. By Lemma 3.4 and Lemma 3.7, we conclude that $\{w, z\} \subseteq S_{1} \backslash\left(\left\{v_{0}\right\} \cup A_{\theta}\left(G \backslash v_{0}\right)\right)$ is a $\theta$-extreme set in $\left(G \backslash v_{0}\right) \backslash A_{\theta}\left(G \backslash v_{0}\right)$. This implies that $\operatorname{mult}\left(\theta,\left(\left(G \backslash v_{0}\right) \backslash A_{\theta}\left(G \backslash v_{0}\right)\right) \backslash w z\right)=3+\left|A_{\theta}\left(G \backslash v_{0}\right)\right|$, contradicting the last sentence of the preceding paragraph. Hence $P_{\theta}(G \backslash v)=\varnothing$ for all $v \in V(G)$ and $G$ is $\theta$-elementary.

Lemma 3.14. Suppose $G$ is $\theta$-elementary. Then for each $\varnothing \neq X \subseteq S \in \mathfrak{P}(\theta, G)$, $A_{\theta}(G \backslash X)=S \backslash X$ and $P_{\theta}(G \backslash X) \cup N_{\theta}(G \backslash X)=\varnothing$.

Proof. Let $x \in X$. Then $P_{\theta}(G \backslash x)=\varnothing$. By Theorem 2.9, $N_{\theta}(G \backslash x)=\varnothing$. Now by Lemma 3.10, $S \backslash\{x\}=A_{\theta}(G \backslash x)$ so that $X \backslash\{x\} \subseteq S \backslash\{x\}=A_{\theta}(G \backslash x)$. By Theorem 1.6, we conclude that $A_{\theta}(G \backslash X)=S \backslash X$ and $P_{\theta}(G \backslash X) \cup N_{\theta}(G \backslash X)=\varnothing$.

Corollary 3.15. Suppose $G$ is $\theta$-elementary. Let $S \subseteq V(G)$. Then $S \in \mathfrak{P}(\theta, G)$ if and only if $G \backslash S$ has exactly $|S|$ components and each is $\theta$-critical.

Proof. Suppose $G \backslash S$ has exactly $|S|$ components and each is $\theta$-critical. Then $c_{\theta}(G \backslash S)=$ $|S|$ and $S$ is a $\theta$-barrier set in $G$. Hence $S \in \mathfrak{P}(\theta, G)$.

The other implication follows from Lemma 3.14 and Corollary 1.8. 


\section{1-elementary cycles}

We shall need the following lemmas.

Lemma 4.1. [10, Corollary 4.4] Suppose $G$ has a Hamiltonian path $P$ and $\theta$ is a root of $\mu(G, x)$. Then every vertex of $G$ which is not $\theta$-essential must be $\theta$-special.

Lemma 4.2. Let $p_{n}$ be a path with $n \geq 1$ vertices. Then

$$
\mu\left(p_{n}, 1\right)=\left\{\begin{array}{lll}
1, & \text { if } n \equiv 0 \text { or } 1 & \bmod 6 \\
-1, & \text { if } n \equiv 3 \text { or } 4 & \bmod 6 \\
0, & \text { otherwise. } &
\end{array}\right.
$$

Proof. Note that for $t \geq 2, \mu\left(p_{t}, x\right)=x \mu\left(p_{t-1}, x\right)-\mu\left(p_{t-2}, x\right)$ (part (c) of Theorem $1.2)$, where we define $\mu\left(p_{0}, x\right)=1$. Therefore $\mu\left(p_{t}, 1\right)=\mu\left(p_{t-1}, 1\right)-\mu\left(p_{t-2}, 1\right)$. Now $\mu\left(p_{1}, 1\right)=1$. So, $\mu\left(p_{2}, 1\right)=0$, and recursively we have $\mu\left(p_{3}, 1\right)=-1, \mu\left(p_{4}, 1\right)=-1$ and $\mu\left(p_{5}, 1\right)=0$. By induction the lemma holds.

Lemma 4.3. Let $C_{n}$ be a cycle with $n \geq 3$ vertices. Then

$$
\mu\left(C_{n}, 1\right)= \begin{cases}1, & \text { if } n \equiv 1 \text { or } 5 \quad \bmod 6 \\ -1, & \text { if } n \equiv 2 \text { or } 4 \quad \bmod 6 \\ 2, & \text { if } n \equiv 0 \quad \bmod 6 ; \\ -2, & \text { if } n \equiv 3 \quad \bmod 6 .\end{cases}
$$

Proof. By part (c) of Theorem 1.2, $\mu\left(C_{n}, 1\right)=\mu\left(p_{n-1}, 1\right)-2 \mu\left(p_{n-2}, 1\right)$. The lemma follows from Lemma 4.2.

Theorem 4.4. A cycle $C_{n}$ is 1-elementary if and only if $n=3 k$ for some $k \in \mathbb{N}$.

Proof. $(\Rightarrow)$ Suppose $C_{n}$ is 1-elementary. Then for any $v \in V\left(C_{n}\right), C_{n} \backslash v=p_{n-1}$. By Lemma $4.2, \operatorname{mult}\left(1, p_{n-1}\right)>0$ if and only if $n-1 \equiv 2$ or $5 \bmod 6$. Thus $n=3 k$ for some $k \in \mathbb{N}$.

$(\Leftarrow)$ Suppose $n=3 k$ for some $k \in \mathbb{N}$. By Lemma 4.3 , $\operatorname{mult}\left(1, C_{n}\right)=0$. Note that $3 k \equiv 3$ or $6 \bmod 6$. Therefore $3 k-1 \equiv 2$ or $5 \bmod 6$, and by Lemma 4.2 and Lemma 1.3, $\operatorname{mult}\left(1, C_{n} \backslash v\right)=\operatorname{mult}\left(1, p_{n-1}\right)=1$ for all $v \in V\left(C_{n}\right)$. Thus $C_{n}$ is 1 -super positive. By Lemma 4.1, $P_{1}\left(C_{n} \backslash v\right)=\varnothing$ for all $v \in V\left(C_{n}\right)$. Hence $C_{n}$ is 1-elementary.

For our next result, let us denote the vertices of $C_{3 k}$ by $1,2,3, \ldots, 3 k$ (see Figure 4 ).

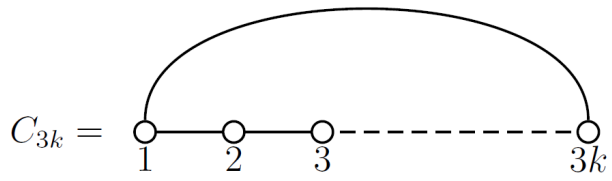

Figure 4. 
Corollary 4.5. $C_{3 k}$ has exactly 3 1-barrier sets, that is

$$
\mathfrak{P}\left(1, C_{3 k}\right)=\{\{1,4,7, \ldots, 3 k-2\},\{2,5,8, \ldots, 3 k-1\},\{3,6,9, \ldots, 3 k\}\} .
$$

Proof. Note that $C_{3 k} \backslash\{1,4,7, \ldots, 3 k-2\}$ is a disjoint union of $k$ number of $K_{2}$ and $K_{2}$ is 1 -critical. So $\{1,4,7, \ldots, 3 k-2\}$ is a 1 -barrier set. Similarly $\{2,5,8, \ldots, 3 k-1\}$ and $\{3,6,9, \ldots, 3 k\}$ are 1-barrier sets. It then follows from Theorem 4.4 and Theorem 3.13 that these are the only 1-barrier sets.

\section{Decomposition of $\theta$-super positive graphs}

Definition 5.1. A set $X \subseteq V(G)$ with $|X|>1$ is said to be independent in $G$ if for all $u, v \in X, u$ and $v$ are not adjacent to each other. A graph $G$ is said to be $\theta$-base if it is $\theta$-super positive and for all $S \in \mathfrak{P}(\theta, G), S$ is independent.

Note that the cycle $C_{3 k}$ is $\theta$-base for $\theta=1$. In fact a connected $\theta$-base graph is $\theta$-elementary.

Theorem 5.2. A connected $\theta$-base graph is $\theta$-elementary.

Proof. Let $G$ be $\theta$-base. Suppose it is not $\theta$-elementary. Then $P_{\theta}(G \backslash v) \neq \varnothing$ for some $v \in V(G)$. By Lemma 2.7, $G \backslash v$ has at least one $\theta$-essential vertex. Moreover, by Theorem 2.9, $N_{\theta}(G \backslash v)=\varnothing$.

If $v$ is not a cut vertex of $G$, then there exists a path from $P_{\theta}(G \backslash v)$ to $D_{\theta}(G \backslash v)$, which implies that $A_{\theta}(G \backslash v) \neq \varnothing$. By Theorem 2.9 and Corollary 1.8, $(G \backslash v) \backslash A_{\theta}(G \backslash v)$ has a $\theta$-super positive component, say $H$. Since $G \backslash v$ is connected, there exists $h \in V(H)$ that is adjacent to some element $w \in A_{\theta}(G \backslash v)$. Note that $\{h, w, v\}$ is a $\theta$-extreme set in $G$. By Lemma 3.4, \{h,w\} is a $\theta$-extreme set in $G$. By Lemma 3.6, $\{h, w\}$ is contained in some $S \in \mathfrak{P}(\theta, G)$, contrary to the fact that $S$ is independent.

If $v$ is a cut vertex of $G$, then either $G \backslash v$ does not have any $\theta$-super positive components or $G \backslash v$ contains a $\theta$-super positive component (recall that $N_{\theta}(G \backslash v)=\varnothing$ ). In the former, $G \backslash v$ must contain a component $H$ such that $A_{\theta}(H) \neq \emptyset$ and $P_{\theta}(H) \neq \emptyset$. Since $H$ is connected, there is a vertex $h \in P_{\theta}(H)$ that is joined to some vertex $w \in A_{\theta}(H)$. Note that $\{h, w, v\}$ is a $\theta$-extreme set in $G$. By Lemma 3.4, $\{h, w\}$ is a $\theta$-extreme set in $G$. By Lemma 3.6, $\{h, w\}$ is contained in some $S \in \mathfrak{P}(\theta, G)$, contrary to the fact that $S$ is independent. In the latter, some vertex in the $\theta$-super positive component, say $u$, must be joined to $v$ so that $\{u, v\}$ is a $\theta$-extreme set in $G$. Again, by Lemma 3.6, $\{u, v\}$ is contained in some $S \in \mathfrak{P}(\theta, G)$, contrary to the fact that $S$ is independent.

Hence $P_{\theta}(G \backslash v)=\varnothing$ for all $v \in V(G)$ and $G$ is $\theta$-elementary.

Note that the converse of Theorem 5.2 is not true. Let $G$ be the graph in Figure 3 . Note that $\left\{u_{3}, u_{4}\right\} \in \mathfrak{P}(1, G)$ but it is not independent. 
Lemma 5.3. Let $G$ be $\theta$-super positive and $e=(u, v) \in E(G)$ such that $\{u, v\}$ is a $\theta$ extreme set in $G$. Let $G^{\prime}$ be the graph obtained by removing the edge e from $G$. Then $G^{\prime}$ is $\theta$-super positive.

Proof. Now mult $(\theta, G \backslash u v)=2$. By part (b) of Theorem 1.2, $\mu(G, x)=\mu\left(G^{\prime}, x\right)-\mu(G \backslash$ $u v, x)$. This implies that $\mu\left(G^{\prime}, \theta\right)=\mu(G, \theta) \neq 0$.

It is left to show that $\mu\left(G^{\prime} \backslash w, \theta\right)=0$ for all $w \in V\left(G^{\prime}\right)$. Clearly if $w=u$ or $v$ then $\mu\left(G^{\prime} \backslash w, \theta\right)=\mu(G \backslash w, \theta)=0$. Suppose $w \neq u, v$. By part (b) of Theorem 1.2 again, $\mu(G \backslash w, x)=\mu\left(G^{\prime} \backslash w, x\right)-\mu(G \backslash w u v, x)$. By Lemma 1.3, mult $(\theta, G \backslash u v w) \geq 1$. Therefore $\mu\left(G^{\prime} \backslash w, \theta\right)=\mu(G \backslash w, \theta)=0$. Hence $G^{\prime}$ is $\theta$-super positive.

Note that after removing an edge from $G$ as in Lemma $5.3, \mathfrak{P}\left(\theta, G^{\prime}\right) \neq \mathfrak{P}(\theta, G)$ in general. In Figure $5, \mathfrak{P}(1, G)=\{\{1,4,7\},\{5,8\},\{6,9\},\{2\},\{3\}\}$. After removing the edge $(1,4)$ from $G$, the resulting graph $G^{\prime}=C_{9}$. By Corollary $4.5, \mathfrak{P}\left(1, G^{\prime}\right)=$ $\{\{1,4,7\},\{2,5,8\},\{3,6,9\}\}$.

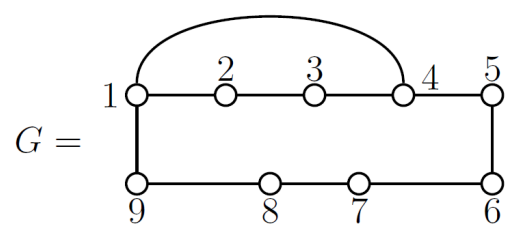

Figure 5.

We shall need the following lemma.

Lemma 5.4. [3, Corollary 2.5] For any root $\theta$ of $\mu(G, x)$ and a path $p$ in $G$,

$$
\operatorname{mult}(\theta, G \backslash p) \geq \operatorname{mult}(\theta, G)-1
$$

Lemma 5.5. Let $G$ be $\theta$-super positive and $e_{1}=(u, v) \in E(G)$ with $\{u, v\}$ is a $\theta$-extreme set. Let $G^{\prime}=G-e_{1}$ and $e_{2}=(w, z) \in E\left(G^{\prime}\right)$. Then $\{w, z\}$ is a $\theta$-extreme set in $G^{\prime}$ if and only if it is a $\theta$-extreme set in $G$.

Proof. Case 1. Suppose $e_{1}$ and $e_{2}$ have a vertex in common, say $w=u$. Then $G^{\prime} \backslash w z=$ $G \backslash w z$.

$(\Rightarrow)$ Suppose $\{w, z\}$ is a $\theta$-extreme set in $G^{\prime}$. By Lemma 5.3 , $\operatorname{mult}\left(\theta, G^{\prime}\right)=0$. Therefore $\operatorname{mult}(\theta, G \backslash w z)=\operatorname{mult}\left(\theta, G^{\prime} \backslash w z\right)=2$ and $\{w, z\}$ is a $\theta$-extreme set in $G$.

$(\Leftarrow)$ The converse is proved similarly.

Case 2. Suppose $e_{1}$ and $e_{2}$ have no vertex in common. By part (b) of Theorem 1.2,

$$
\mu(G \backslash w z, x)=\mu\left(G^{\prime} \backslash w z, x\right)-\mu(G \backslash w z u v, x) .
$$


$(\Rightarrow)$ Suppose $\{w, z\}$ is a $\theta$-extreme set in $G^{\prime}$. Then $\operatorname{mult}\left(\theta, G^{\prime} \backslash w z\right)=2$. Now $\operatorname{mult}(\theta, G \backslash u v)=2$ and by Lemma $5.4, \operatorname{mult}(\theta, G \backslash u v w z) \geq 1$. So we conclude that $\operatorname{mult}(\theta, G \backslash w z) \geq 1$. On the other hand, $N_{\theta}(G \backslash w)=\varnothing$ (Theorem 2.9). Therefore either $\operatorname{mult}(\theta, G \backslash w z)=0$ or 2 . Hence the latter holds and $\{w, z\}$ is a $\theta$-extreme set in $G$.

$(\Leftarrow)$ Suppose $\{w, z\}$ is a $\theta$-extreme set in $G$. Then $\operatorname{mult}(\theta, G \backslash w z)=2$. As before we have $\operatorname{mult}(\theta, G \backslash u v w z) \geq 1$. So we conclude that $\operatorname{mult}\left(\theta, G^{\prime} \backslash w z\right) \geq 1$. On the other hand, by Lemma 5.3, $G^{\prime}$ is $\theta$-super positive. Therefore $N_{\theta}\left(G^{\prime} \backslash w\right)=\varnothing$ (Theorem 2.9), and then either mult $\left(\theta, G^{\prime} \backslash w z\right)=0$ or 2 . Hence the latter holds and $\{w, z\}$ is a $\theta$-extreme set in $G^{\prime}$.

Definition 5.6. Let $G$ be $\theta$-super positive. An edge $e=(u, v) \in E(G)$ is said to be $\theta$-extreme in $G$ if $\{u, v\}$ is a $\theta$-extreme set.

The process described in Lemma 5.3, can be iterated. Let $Y_{0}=\left\{e_{1}, e_{2}, \ldots, e_{k}\right\} \subseteq E(G)$ be the set of all $\theta$-extreme edges. Let $G_{1}=G-e_{1}$. Then $G_{1}$ is $\theta$-super positive (Lemma 5.3). Let $Y_{1}$ be the set of all $\theta$-extreme edges in $G_{1}$. Then by Lemma 5.5, $Y_{1}=Y_{0} \backslash\left\{e_{1}\right\}$. Now let $G_{2}=G_{1}-e_{2}$. By applying Lemma 5.3 and Lemma 5.5 , we see that $G_{2}$ is $\theta$-super positive and the set of all $\theta$-extreme edges in $G_{2}$ is $Y_{2}=Y_{0} \backslash\left\{e_{1}, e_{2}\right\}$. By continuing this process, after $k$ steps, we see that $G_{k}=G-e_{1} e_{2} \ldots e_{k}$ is $\theta$-super positive and the set of all $\theta$-extreme edges in $G_{k}$ is $Y_{k}=\varnothing$. We claim that $G_{k}$ is a disjoint union of $\theta$-base graphs. Suppose the contrary. Let $H$ be a component of $G_{k}$ that is not $\theta$-base. Since $G_{k}$ is $\theta$-super positive, by part (a) of Theorem 1.2, we deduce that $H$ is $\theta$-super positive. Therefore there is a $S \in \mathfrak{P}(\theta, H)$ for which $S$ is not independent. Let $e=(u, v) \in E(H)$ with $\{u, v\} \subseteq S$. By Lemma 3.7 and Lemma 3.4, $\{u, v\}$ is a $\theta$-extreme set in $H$. This means that $e$ is $\theta$-extreme in $H$, and by part (a) of Theorem 1.2,e is $\theta$-extreme in $G_{k}$, contrary to the fact that $Y_{k}=\varnothing$. Hence $H$ is $\theta$-base and we have proved the following theorem.

Theorem 5.7. Let $G$ be $\theta$-super positive. Then $G$ can be decomposed into a disjoint union of $\theta$-base graphs by deleting its $\theta$-extreme edges. Furthermore, the decomposition is unique, i.e. the $\theta$-base graphs are uniquely determined by $G$.

The proof of the next lemma is similar to Lemma 5.3, and is thus omitted.

Lemma 5.8. Let $G$ be $\theta$-super positive and $\{u, v\}$ is a $\theta$-extreme set with $e=(u, v) \notin$ $E(G)$. Let $G^{\prime}$ be the graph obtained by adding the edge e to $G$. Then $G^{\prime}$ is $\theta$-super positive.

Using the process described in Lemma 5.8, we can construct $\theta$-super positive graph from $\theta$-base graphs. Together with Theorem 5.7, we see that every $\theta$-super positive can be constructed from $\theta$-base graphs.

Corollary 5.9. A graph is $\theta$-super positive if and only if it can be constructed from $\theta$-base graphs. 
In the next theorem, we shall extend Theorem 2.2.

Theorem 5.10. Let $G_{1}$ and $G_{2}$ be two $\theta$-super positive graphs and $S_{i} \in \mathfrak{P}\left(\theta, G_{i}\right)$ for $i=1,2$. Let $G$ be the graph obtained by adding the edges $e_{1}, e_{2}, \ldots, e_{m}$ to the union of $G_{1}$ and $G_{2}$, where each $e_{j}$ contains a point in $S_{1}$ and $S_{2}$. Then $G$ is $\theta$-super positive.

Proof. We shall prove by induction on $m$. If $m=1$, we are done by Theorem 2.2. Suppose $m \geq 2$. Assume that it is true for $m-1$. Let $G^{\prime}$ be the graph obtained by adding the edges $e_{1}, e_{2}, \ldots, e_{m-1}$ to the union of $G_{1}$ and $G_{2}$. By induction $G^{\prime}$ is $\theta$-super positive. Let $e_{m}=\left(v_{1}, v_{2}\right)$ where $v_{i} \in S_{i}$. Note that the number of $\theta$-critical components in $G^{\prime} \backslash\left(S_{1} \cup S_{2}\right)$ is $c_{\theta}\left(G^{\prime} \backslash\left(S_{1} \cup S_{2}\right)\right)=c_{\theta}\left(G_{1} \backslash S_{1}\right)+c_{\theta}\left(G_{2} \backslash S_{2}\right)=\left|S_{1}\right|+\left|S_{2}\right|$. So $S_{1} \cup S_{2}$ is a $\theta$-barrier set in $G^{\prime}$. By Lemma 3.7 and Lemma 3.4, $\left\{v_{1}, v_{2}\right\}$ is a $\theta$-extreme set in $G^{\prime}$. Therefore by Lemma 5.8, $G$ is $\theta$-super positive.

In Figure 6, the graph $G$ is obtained from two 1-base graphs by adding edges $e_{1}$ and $e_{2}$.

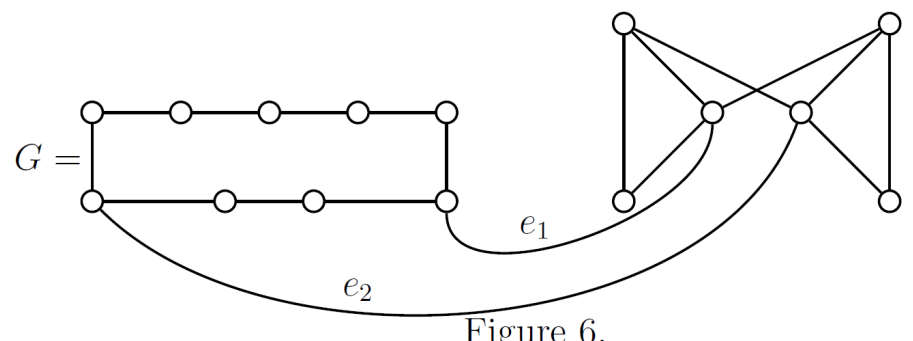

\section{Acknowledgment}

We would like to thank the anonymous referee for the comments that helped us make several improvements to this paper.

\section{References}

[1] W. Chen and C. Y. Ku, An analogue of the Gallai-Edmonds structure theorem for nonzero roots of the matching polynomial, Journal of Combinatorial Theory Series B 100 (2010), 119-127.

[2] C. D. Godsil, Algebraic Combinatorics, Chapman and Hall, New York (1993).

[3] C. D. Godsil, Algebraic matching theory, The Electronic Journal of Combinatorics 2 (1995), \# R8. 
[4] O. J. Heilmann and E. H. Lieb, Theory of monomer-dimer system, Commun. Math. Physics 25 (1972), 190-232.

[5] C. Y. Ku and K. B. Wong, Extensions of barrier sets to nonzero roots of the matching polynomial, Discrete Mathematics 310 (2010), 3544-3550.

[6] C. Y. Ku and K. B. Wong, Gallai-Edmonds structure theorem for weighted matching polynomial, available at http://arxiv.org/abs/1006.1028.

[7] C. Y. Ku and K. B. Wong, Generalized D-graphs for nonzero roots of the matching polynomial, Discrete Mathematics 311 (2011), 2174-2186.

[8] C. Y. Ku and K. B. Wong, Generalizing Tutte's theorem and saturated nonfactorizable graphs, submitted.

[9] C. Y. Ku and K. B. Wong, Maximum multiplicity of a root of the matching polynomial of a tree and minimum path cover, The Electronic Journal of Combinatorics 16 (2009), \#R81.

[10] C. Y. Ku and K. B. Wong, Maximum multiplicity of matching polynomial roots and minimum path cover in general graph, The Electronic Journal of Combinatorics $\mathbf{1 8}$ (2011), \#P38.

[11] L. Lovász and M. D. Plummer, Matching Theory, Elsevier Science Publishers, Budapest (1986). 